\title{
Towards a technology-based assessment of sensory-motor pathological states through tactile illusions
}

\author{
Gemma Carolina Bettelani ${ }^{1 \dagger}$, Alessandro Moscatelli ${ }^{2}$, and Matteo Bianchi ${ }^{1}$
}

\begin{abstract}
Touch provides important information on the physical properties of external objects, and contributes to the sense of our hand position and displacement in perceptual tasks. Recent studies showed that the texture of the touched surface produced a bias on the perceived tactile motion, ultimately affecting the direction of hand motion in reaching tasks. Specifically, moving on a plate with parallel ridges, the hand motion deviates towards a direction opposite with respect to the one predicted by tactile flow mathematical model, i.e. perpendicular to the ridges. Here, we used this phenomenon to quantitatively assess an impairment in tactile channel. We asked healthy participants slide the hand on a plate with parallel ridges, either with bare fingertip or by wearing a glove. The glove condition simulated a dysfunction in tactile channel, as may occur in pathological conditions, for e.g. due to a neurological disease. Our hypothesis is that, wearing a glove, the systematic error induced by the texture orientation will be smaller because the information provided by the tactile channel is noisier. Results are in agreement with our hypothesis, and could open interesting perspectives towards a quantitative technology-based tool for the assessment of tactile impairment in pathological conditions.
\end{abstract}

\section{INTRODUCTION}

Touch provides important information on the physical properties of external objects, and contributes to the sense of our hand position and displacement [1][2]. In particular, it was shown that tactual interaction with external objects contributes to our internal representation of hand position and motion, that is, to proprioception [3], [4], [5], [6], [7], [8]. Our recent studies showed that the deformation of the fingertip's skin occurring when we push the finger against a soft surface generates the illusory sensation of finger displacement [9]. In [10] we showed that touch provides also auxiliary cues to guide hand displacement. In this previous study, we asked the blindfolded participant to contact a plate with parallel ridges and to move straight ahead. Participants produced a systematic error in the hand trajectory, with the hand motion deviating towards the major axis of the ridges (i.e. in a direction opposite to the one predicted by tactile flow model, which is perpendicular to the ridges [11]). We provided the following explanation of the results. When

${ }^{1}$ G. C. Bettelani and M. Bianchi are with Centro di Ricerca "E. Piaggio", Università di Pisa, Pisa, Italy and with the Department of Information Engineering of Università di Pisa, Pisa, Italy. \{m.bianchi\} at centropiaggio.unipi.it, \{gemma.bettelanil\} at gmail.com

${ }^{2}$ A. Moscatelli is with Department of Systems Medicine and Centre of Space Bio-medicine, University of Rome "Tor Vergata", Rome, Italy and with the Laboratory of Neuromotor Physiology, IRCCS Santa Lucia Foundation, Rome, Italy. $\{a \cdot$ moscateli $\}$ at hstantalucia.it

$\dagger$ Corresponding author. sliding the fingertip on the plate, the perceived direction of the tactile motion was perpendicular to the ridges, because motion component parallel to the ridges does not produce a relevant change in tissue strain as predicted by the tactile flow mathematical model [11]. Hence, participants corrected the movement, in a direction opposite to the perceived tactile motion. This support the hypothesis that the perceived hand displacement arises from a weighted sum of classical proprioception cues (i.e. due to muscular-skeletal system, unbiased) and touch (Fig. 1). Here, we aimed to exploit this phenomenon to quantitatively assess an impairment in tactile channel, as for example a reduced sensitivity.

Several diseases affecting the nervous system-including stroke, multiple sclerosis (MS) and diabetic neuropathy, to mention some of the most common-may cause dysfunctions in cutaneous touch, such as paresthesia (abnormal sensation such as tingling or tickling) and hypoestesia (reduced tactile sensitivity) [12]. For example, sensory deficits including hypo- and paraesthesia occur approximately in $85 \%$ of patients affected by multiple sclerosis (MS) [13]. In clinical practice, simple diagnostic procedures are used to test tactile dysfunctions in neurological patients. These include twopoint discrimination (for e.g. using von Frey hairs), detection of skin vibrations, detection of light stroke with a cotton wisp [12]. The assessment performed by means of these clinical tests is scarcely reproducible, because the delivery of the stimuli does not involve the usage of mechatronic devices. In this study, we proposes a proof-of-concept method for automatic detection of tactile impairment, which is based on well established results in behavioral neuroscience [14], [10]. The ambitious long-term objective of this work is pave the path towards a reproducible clinical tests to measure tactile dysfunction in neurological patients.

We tested the hypothesis that, sliding our hand covered by a glove, on a surface with ridges in accordance with the one used in [10], the bias of the hand trajectories with respect to the straight path should be smaller with respect to the case of bare finger sliding. Indeed, while wearing a glove, the sense of touch is attenuated. This situation simulates the case of a subject affected by hypoestesia. Thus, we suggest that in our tested conditions the model proposed in [10], where the perceived direction of hand motion is equal to a weighted sum of the direction estimates by the classical proprioception (e.g. from receptors in the musculoskeletal system) and the one estimates by the tactile slip, still remains valid, but the contribution of the touch is less substantial. For this reason, the perceived movement of the hand should be less biased toward a direction perpendicular to the orientated 


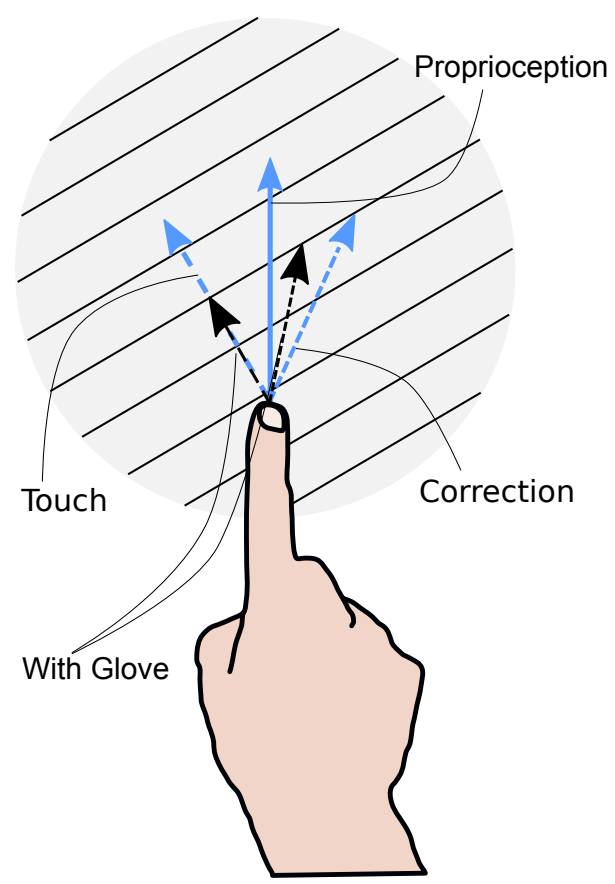

Fig. 1: When a subject wants to move the hand on a plate with oblique ridges, along the direction indicated by the solid arrow (desidered path), the cutaneous feedback produces an illusory sensation of bending towards a direction perpendicular to the ridges (dashed arrow). This effect is in accordance with the tactile flow model [14]. This eventually led to an adjustment of the motion trajectory towards a direction opposite to the one of tactile flow (dotted arrow), so there is an angular deviation from the desidered path. In case of reduced tactile sensation (in black), the weight of the effect of tactile flow in hand perception is reduced, thus the resulting bias (i.e. deviation from the desidered path) is inferior with respect to the bare finger case. Note that the actual hand trajectory also depend on extra-cutaneous cues, e.g. from musculoskeletal system (not shown in the picture) [10].

ridges, leading to a smaller deviation of the hand trajectory in a direction opposite to the tactile slip.

Experimental results of this work are in agreement with our hypothesis and open interesting perspectives towards the development of mechatronic devices for the quantification of tactile impairment. Indeed, the lower will be the bias in the direction of hand motion, the greater will be the deficit in the sense of touch. In future applications, the current results can have an important impact for the assessment of tactile dysfunction in neurological disease, such as peripheral neuropathy in diabetic patients, hereditary neuropathy, traumatic nerve injuries and other inflammatory diseases of the Central Nervous System (CNS) including MS.

\section{METHODS}

\section{A. Participants}

Seven naive healthy participants took part in the experiment (1 male and 6 females, age: $26.6 \pm 1.1$, mean \pm standard deviation). Participants were all right-handed and reported no medical conditions that could have affected the experimental results. The testing procedures were approved by the Ethical Committee of the University of Pisa, in accordance with the guidelines of the Declaration of Helsinki for research involving human subjects. Informed consent was obtained from all participants involved in the study.

\section{B. Experimental setup}

For our experiment, we have reproduced the same experimental set-up of [10]. The set-up (Fig. 2) included a circular plate with a load cell (Micro Load Cell, 0 to 780 g, CZL616C from Phidgets, Calgary, AB-Canada) to record the normal contact forces, and over this plate, we placed a circular surface (diameter of $15 \mathrm{~cm}$ ) with parallel ridges. The ridges had a square section of $1 \mathrm{~mm} \times 1 \mathrm{~mm}$ and their spacial frequency was $1 \mathrm{~cm}$, consistently with [11]. A Leap Motion device (Leap Motion Inc., San Francisco, U.S.) was placed above the surface in face down configuration for the tracking of the index finger. The sampling frequency of the Leap Motion is $40 \mathrm{~Hz}$.

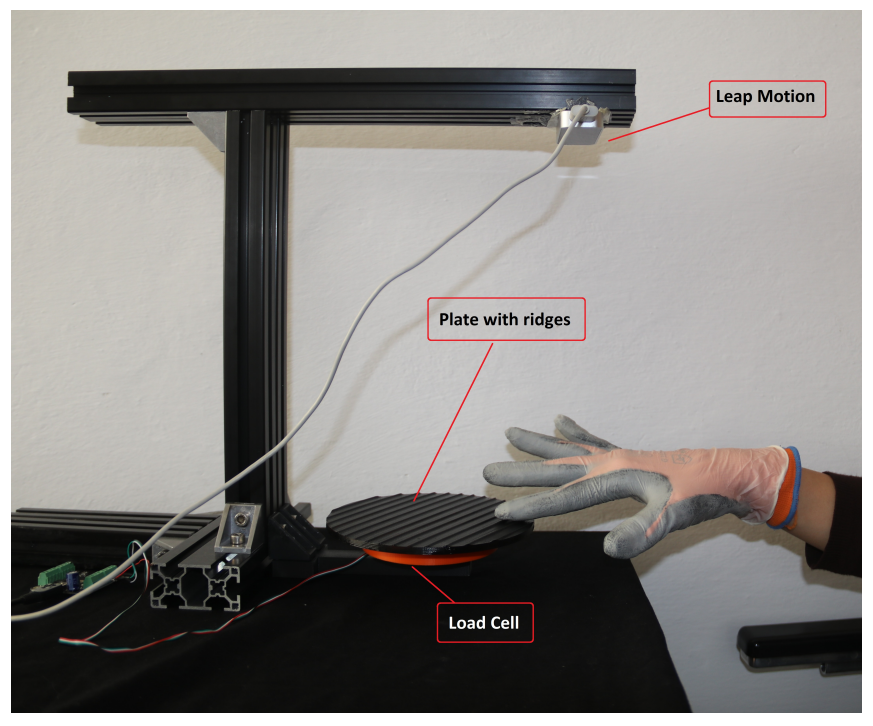

Fig. 2: The experimental set-up including the plate with ridges, the Phidgets Micro Load Cell and the Leap Motion device. In this trial the participant wears the glove

\section{Protocol of the experiment}

The participants were sitting on an office chair in front of the set-up. They were blindfolded and were isolated from external feedback, wearing headphones playing pink noise. For each trial, the subjects were instructed to perform a straight path, going ahead with their right index finger for approximately $10 \mathrm{~cm}$, as in [10]. Participants had to keep the fingertip in contact with the plate for the entire duration of the trial, and they were instructed, during some preparatory trials, to apply a level of normal force less than $2 \mathrm{~N}$ for the entire duration of the experiment as in [10]. After the experiment, we performed a check on the force values to verify the fulfillment of force constrains. Furthermore, before the onset of the experimental trials, we also verified the participants were able to feel the ridges, while wearing the glove too.

The plate were rotated by the experimenter before each trial in order to obtain different ridges orientation: -60, 30, 0, 30, $60 \mathrm{deg}$ (Fig. 3). A zero angle means that the ridges of the plate were parallel to the frontal plane of the participant, this means that were perpendicular to the finger's movement, whereas negative (positive) angles means that the ridges were rotated clockwise (counterclockwise). 
Each stimulus orientation was presented ten times in pseudorandom order, for a total of fifty stimuli. The subjects performed two experimental sessions, one wearing a "glove" (i.e. a working glove with soft pads and thickness $1 \mathrm{~mm}$ - Ta-Ku G332 - and two latex gloves) and the other one without. We chose this "glove" without any clinical claim and after preliminary experiments, which aimed at finding the right solution that produced sensory impairment, still retaining subjects' capabilities to perform the task and feel the ridges. The objective was to provide a proof of concept to demonstrate the effect of reduced tactile impairment in tactile slip-mediated hand perception. The order of the two sessions was randomized. Participants received no feedback about their performance during the experiment. At the end of each trial, the experimenter lifted the hand of the participant to place it back to the starting position, taking care that the forearm was aligned with the finger.

\section{Data Analysis}

The trajectory of the index finger was recorded with the Leap Motion device and saved for the analysis. The angular deviation from a straight-ahead motion direction (e.g., the deviation from the direction given by the solid arrow in Fig. 1) was computed using the $\arctan \left(\frac{y}{x}\right)$, where $x$ and $y$ are the coordinates of the final positions of the index finger. When the angles are negative (positive) means that the subject performed, with the index finger, a trajectory deviated toward right (left) with respect to the straight path (meaning no bias). Linear Mixed Model (LMM) was used to evaluate the effect of the orientation of the ridges $(\mathbf{X})$ on the angular deviation from the straight direction (A), and how the presence of the glove modulated the phenomenon (interaction between ridges and glove):

$$
\mathbf{A}=\beta_{0}+u_{0}+\left(\beta_{1}+u_{1}\right) \mathbf{X}+\varepsilon,
$$

where $\beta_{0}$ and $\beta_{1}$ are the fixed- effect intercept and slope, respectively, $u_{0}$ and $u_{1}$ are the random-effect intercept and slope of the model (between-participant), and $\varepsilon$ is the residual error term.

Data analysis was performed in $\mathrm{R}(\mathrm{R}$ version 3.3.2) and the R Package $\operatorname{lm} 4$ was used to fit LMM [15]. In LMM, we tested the significance of the slope parameter $\beta_{1}$, by means of the Likelihood Ratio Test.

\section{RESUlts}

Fig. 5 shows the angular deviation of the hand trajectory from the mid-line with respect to the orientation of the ridges, for participants P01-P07.

In accordance with [10], when participants did not wear the glove, the hand trajectory is deviated toward a direction parallel to the ridges (effect size: $-0.19 \pm 0.04, \beta_{1} \pm$ Std.
TABLE I: Average angular deviations from the straight path

\begin{tabular}{ccc}
\hline Stimulus & Angular error (no glove) & Angular error (glove) \\
\hline$-30^{\circ}$ & $23.5^{\circ}$ & $0.9^{\circ}$ \\
$-60^{\circ}$ & $25.5^{\circ}$ & $7.3^{\circ}$ \\
$30^{\circ}$ & $-4.8^{\circ}$ & $-1.2^{\circ}$ \\
$60^{\circ}$ & $-9.2^{\circ}$ & $-1.1^{\circ}$ \\
\hline
\end{tabular}

Error). In particular, there is a deviation of the hand motion toward left (positive values of $y$ ) for a clockwise rotation and vice-versa.

Moreover, when participants wore the glove, the trend of the hand motion trajectories remains the same (Fig. 4) as in the case in which they did not wear the glove, but the bias from the mid-line of the plate, due to the effect tactile flow [11] and hence to the tactile input, is smaller. In fact, the effect size is reduced and the interaction between ridges and glove is statistically significant (effect size: $0.12 \pm 0.04$ ). Accordingly, as illustrated in Fig. 6, the slope of the linear relationship between the angular error and the ridges was significantly more negative without the glove than with it $\left(\chi_{1}=4.4, \mathrm{p}=0.03\right)$.

Fig. 4 shows five trajectories, one for each stimulus, of participant P02. The trajectories performed without the glove (on the top) are characterized by higher deviation from the mid-line with respect to the trajectories performed with the glove (on the bottom).

In addition to this, the Table 1 shows the average angular deviations from the mid-line in the two cases for one exemplary participant (P02).

What is noticeable is that the average angular deviation from the mid-line of the plate is considerably reduced when the participant wore the glove.

Furthermore, as it is shown in Fig. 5, there is a significant offset in the linear function with larger absolute bias for clockwise rotation stimuli; this is the reason why at $0^{\circ}$ the trajectories are not perfectly straight. This effect is in accordance with [10] and represents a systematic error, due to extra-cutaneous cues, called haptic oblique effect, as explained in [16]. The effect is present in both experiments and accounted for the asymmetry of the results between the clock- and the counterclockwise rotation of the stimuli (Fig. 5 and Fig. 4).

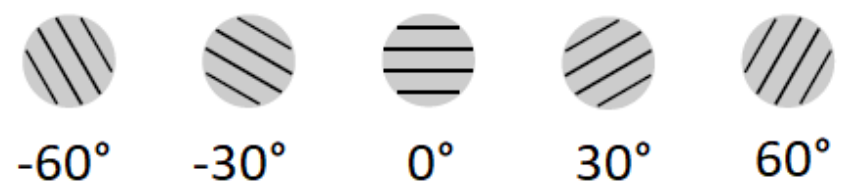

Fig. 3: Ridges orientation 

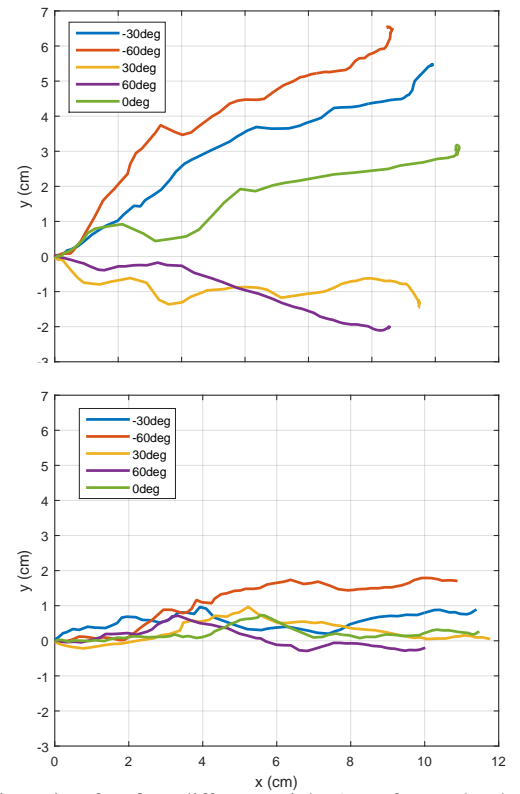

Fig. 4: The trajectories for five different trials (one for each plate orientation) of participant P02. Positive y values are for a leftward deviation from the mid-line, while negative y values are for rightward deviation. In the top panel, the trajectories refer to trial executed without the glove, while in the bottom panel with the glove

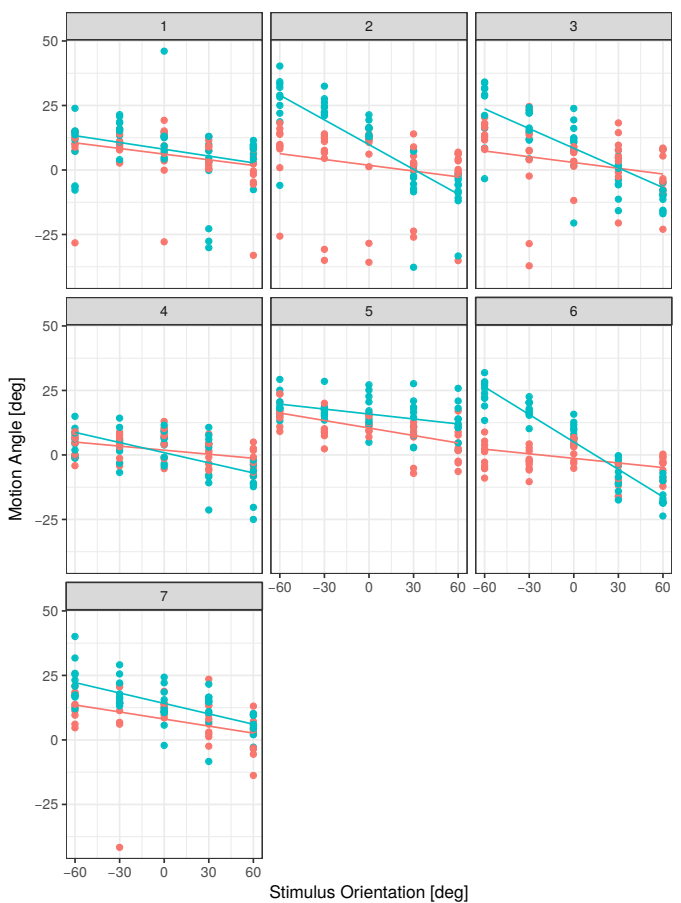

Fig. 5: The angular deviation from the mid-line of the hand trajectories with respect to the ridges orientation for participants P01-P07. The point data represents the angular deviation for a single trial and the line the LMM prediction. In red results for trials with the glove, in green results without the glove.

\section{DiscusSiON AND FUTURE WORKS}

In previous works, we demonstrated that tactile slip contributed to hand displacement inducing a bias in hand position perception, while the subjects were instructed to slide with their index finger on a ridged plate and to produce a straight path [10]. More specifically, we observed a perceptual bias explained by tactile flow model (i.e. which
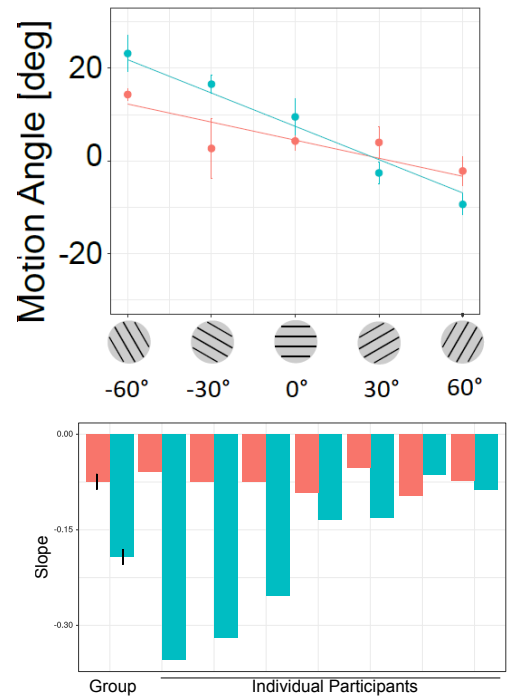

Fig. 6: On the top the angular error of the hand trajectory with respect to the mid-line of the plate regressed against the orientation of the ridges. Positive y values are for a leftward deviation from the mid-line and negative $y$ values are for a rightward deviation. In accordance with [10] when the participant did not wear the glove, there is a negative relationship (negative slope) between the angular deviation and the ridges orientation (green line), and this relationship is significantly less negative when participants wore the glove (i.e. the bias induced by tactile flow is reduced) than without it (red line). Data are linear fitted for a representative participant (P06). On the bottom, the slope of the linear relationship for 7 participants with group estimate and standard deviation (LMM estimates)

elicits a perceived direction perpendicular to the ridges), which triggered a corrective action in the opposite direction. We found that the resulting hand perception was a weighted sum of tactile and classical proprioception (i.e. related to musculo-skeletal system). In this work, we pushed further this investigation, and tested the hypothesis that an impairment of cutaneous touch, as it is provoked by a glove, could result in a reduced bias. We performed experiments with and without tactile impairment, finding that when a subject wore a glove the bias was statistically minor than the one in the bare finger conditions.

We could potentially consider the alternative explanation that the angular error was due to the effect of the frictional force exerted by the ridges, rather than to the neural mechanism we hypothesized. However, two considerations support our hypothesis that the motor error arises from motor control, rather than mechanical factors. First, the tangential force due to texture geometry and friction is smaller for a motion direction parallel to the ridges: Hence, if this tangential force produced the bias, the motor error should be larger at $\pm 30^{\circ}$ than at $\pm 60^{\circ}$, which is the opposite of what we found. Furthermore, in passive touch, it was showed that the orientation of the ridges biased the perceived surface motion also when the frictional force was controlled through lubrication of the sliding surface [14], or when tactile motion was given by a pin display that did not produced tangential friction [17]. For the reason expressed above, we can reasonably conclude that the angular error is due to the effect of the perceived tactile motion and not to the frictional force. Further investigations will be performed to strengthen these conclusions.

The outcomes of the paper opens interesting perspective 
for the development of quantitative tools to assess tactile impairment, which is a common symptom in several neurological diseases. In other terms, the greater the damage to tactile sensory apparatus, the less the bias. Future works will be devoted to apply the here reported methods to clinical evaluation of neurological patients, paving the path towards the design of compact mechatronic devices that can be used to provide reliable and quantitative estimation of tactile dysfunction and evaluate the outcomes of rehabilitative procedures. Other analysis will be also performed to compute tactile contribution to hand position perception with patients. Applications to targeted pathological populations, such as peripheral neuropathy in diabetic patients, hereditary neuropathy, traumatic nerve injuries, and inflammatory diseases of the CNS such as MS will be considered.

\section{ACKNOWLEDGMENTS}

This research has received funding from the European Union's Horizon 2020 Research and Innovation Programme under Grant Agreement No.688857 (SoftPro). The content of this publication is the sole responsibility of the authors. The European Commission or its services cannot be held responsible for any use that may be made of the information it contains. Authors would like to thank Simone Ciotti, Giuseppe Averta, Davide Doria and Edoardo Battaglia for their support in building up the experimental setup.

\section{REFERENCES}

[1] S. J. BensmaIa and M. Hollins, "The vibrations of texture." Somatosensory \& motor research, vol. 20, no. 1, pp. 33-43, jan 2003.

[2] R. Johansson and G. Westling, "Roles of glabrous skin receptors and sensorimotor memory in automatic control of precision grip when lifting rougher or more slippery objects," Experimental Brain Research, vol. 56, no. 3, pp. 550-564, oct 1984.

[3] H. C. Bastian, "The 'muscular sense': its nature and cortical localisation," Brain, vol. 10, pp. 1-137, 1888.

[4] B. B. Edin and J. H. Abbs, "Finger movement responses of cutaneous mechanoreceptors in the dorsal skin of the human hand," J Neurophysiology, vol. 65, no. 3, pp. 657-670, 1991.

[5] D. F. Collins, K. M. Refshauge, G. Todd, and S. C. Gandevia, "Cutaneous receptors contribute to kinaesthesia at the index finger, elbow and knee," J Neurophysiology, vol. 94, pp. 1699-1706, 2005.

[6] N. S. Weerakkody, D. A. Mahns, J. L. Taylor, and G. S. C, "Impairment of human proprioception by high-frequency cutaneous vibration," J Physiology, vol. 581, pp. 971-980, 2007.

[7] U. Proske and S. C. Gandevia, "The proprioceptive senses: their roles in signaling body shape, body position and movement, and muscle force," Physiological reviews, vol. 92, no. 4, pp. 1651-1697, 2012.

[8] B. B. Edin and N. Johansson, "Skin strain patterns provide kinaesthetic information to the human central nervous system." The Journal of physiology, vol. 487, no. 1, pp. 243-251, 1995.

[9] A. Moscatelli, M. Bianchi, A. Serio, A. Terekhov, V. Hayward, M. O. Ernst, and A. Bicchi, "The change in fingertip contact area as a novel proprioceptive cue," Current Biology, vol. 26, no. 9, pp. 1159-1163, 2016.

[10] M. Bianchi, A. Moscatelli, S. Ciotti, G. C. Bettelani, F. Fioretti, F. Lacquaniti, and A. Bicchi, "Tactile slip and hand displacement: Bending hand motion with tactile illusions," in World Haptics Conference (WHC), 2017 IEEE. IEEE, 2017, pp. 96-100.

[11] A. Bicchi, E. P. Scilingo, E. Ricciardi, and P. Pietrini, "Tactile flow explains haptic counterparts of common visual illusions," Brain Res Bull., vol. 75, no. 6, pp. 737-741, 2008.

[12] E. R. Kandel, J. Schwartz, T. Jessel, S. A. Siegelbaum, and A. J. Hudspeth, Principles of Neural Science, E. R. Kandel, J. Schwartz, T. Jessel, S. A. Siegelbaum, and A. J. Hudspeth, Eds. New York, NY: McGraw-Hill, 2013.
[13] T. Vos, C. Allen, M. Arora, R. M. Barber, Z. A. Bhutta, A. Brown, A. Carter, D. C. Casey, F. J. Charlson, A. Z. Chen, et al., "Global, regional, and national incidence, prevalence, and years lived with disability for 310 diseases and injuries, 1990-2015: a systematic analysis for the global burden of disease study 2015," The Lancet vol. 388, no. 10053, pp. 1545-1602, 2016.

[14] A. Bicchi, D. E. De Rossi, and E. P. Scilingo, "The role of the contact area spread rate in haptic discrimination of softness," IEEE trans. on Robotics and Automation, vol. 16, no. 5, pp. 496-504, October 2000.

[15] D. Bates, M. Mächler, B. Bolker, and S. Walker, "Fitting Linear Mixed-Effects Models Using lme4," Journal of Statistical Software, vol. 67 , no. 1 , pp. $1-51$, jun 2015

[16] A. M. L. Kappers and J. J. Koenderink, "Haptic perception of spatial relations," Perception, vol. 28, no. 6, pp. 781-795, 1999.

[17] Y.-C. Pei, S. S. Hsiao, and S. J. Bensmaia, "The tactile integration of local motion cues is analogous to its visual counterpart," Proceedings of the National Academy of Sciences, vol. 105, no. 23, pp. 8130-8135, 2008. 